\title{
URBAN MOBILITY AND INFLUENCE FACTORS: A CASE STUDY OF PRAGUE
}

\author{
LADISLAVA FIALKA SOBKOVÁ ${ }^{1} \&$ MICHAL ČERTICKÝ ${ }^{2}$ \\ ${ }^{1}$ Czech Technical University in Prague, Faculty of Architecture, MOLAB, Czech Republic \\ ${ }^{2}$ Czech Technical University in Prague, Faculty of Electrical Engineering, \\ Department of Computer Science, Czech Republic
}

\begin{abstract}
Walking is the most natural human movement. A lack of walking on a daily basis causes a number of lifestyle diseases including diabetes, obesity and cardiovascular diseases. These medical complications are increasingly widespread by urban lifestyle. Comfort of non-physical activity allowed by developed transportation systems leads to the pandemic of physical passivity. Our case study is based on the data set of daily activity schedules of 89,948 urban citizens extracted from agent-based simulation model of multimodal mobility of Prague. The schedules contain the exact routes, transport modes and durations of all the trips made by the public transport users. The analysis proves that most of the walking trajectory is composed of the necessary daily routine: walk from home to the public transport station, walk from a different station to the workplace and back. This trajectory covers on average $85.4 \%$ of daily walking distance. With increasing age, the percentage is slightly higher. An average inhabitant of Prague, Czech Republic walks $3.1 \mathrm{~km}$ per day, which is considered a low daily physical activity. Residents are considered active, if they walk more than $6.6 \mathrm{~km}$ on an average day. We did not find a statistically relevant correlation with the marital status or education. However, a correlation with financial income is apparent: an average walking distance is higher in households with income higher than 1,130 EUR per household member. That could be caused by the fact that higher income Prague families tend to reside in the areas with lower building density and worse public transport connectivity. The daily travelling routine constitutes a majority of daily physical movement, which seems to be insufficient at the moment.
\end{abstract}

Keywords: walking, public space, transport, agent-based modeling, activity-based modeling, public health.

\section{INTRODUCTION}

An approach to city development has changed significantly during the last century [1]. Functional cities with strictly divided zones for work, housing and leisure time, defined by Athens charter in 1940s [2], were questioned by Jane Jacobs in early 1970s [3]. She started to introduce sociological concepts such as "eyes on the street" and "social capital" to the city development. This shifted the focus on the pedestrians and their movement.

The society also came through several changes and is facing new threats: at the beginning of the $20^{\text {th }}$ century, people in overpopulated cities were affected by bacterial diseases such as cholera and tuberculosis. Nowadays, civilizational diseases are the main healthcare issue. Stress, lack of physical activity on a daily basis and desk work are the main risk factors for the cardiovascular diseases (CVD), cancer, and diabetes. Pandemic of inactivity [4] puts those diseases (CVD, cancer) on the top of the healthcare statistics [5], [6]. 31\% of world population is not meeting even the minimal requirements for physical activity [4]. Regular walking more than 10,000 steps per day is proven to lower the blood pressure and improve physical condition [7], [8]. This basic physical activity has positive health consequences for people of all age groups [9]. 


\subsection{Economy of pedestrians}

Walking is the basic human movement and is crucial part of mobility in the cities [10]. The benefits for the pedestrians are not just in the category of "public health". There are also social, environmental, and economic benefits. The turnover in middle European and Scandinavian city centers increased in $60 \%$ and remained constant in $25 \%$ after being pedestrianized [11].

\subsection{Local conditions}

The data about the population of Prague, Czech Republic in 2013 indicate, that $42 \%$ of mortality rate is caused by cardiovascular diseases among women and $49 \%$ among men [6]. The rate of CVD mortality is slightly decreasing in Czech population, but CVD occurrence is still significantly higher than in Western Europe [5]. Lack of physical activity is obvious even in the childhood - the percentage of overweight children has increased since the $90 \mathrm{~s}$, and so did the BMI of the parents [12]. Percentage of overweight and obese fathers is continuously increasing from $50 \mathrm{~s}$. Overweight of the children is probably also caused by bigger income of their parents and different nutrition possibilities [21].

Prague has developed and dense system of public transport. Average distance of inhabited address from the public transport stop is $257 \mathrm{~m}$. This density is for sure comfortable for the citizens, but on the other hand is not forcing the habitants to the regular physical activity.

\subsection{Impact of city infrastructure}

Psychological and health influence of the relationship between the environment and the pedestrian movement has already been examined by previous studies [12], [13]. This paper focuses on narrower segment of the city - the transport infrastructure and its influence on pedestrian activity in the city. The influence of the public transport accessibility on daily physical activity of residents could be considered an important factor in the city development.

\section{METHOD OF THE RESEARCH}

For our study, we decided to use the data from agent-based simulation model of mobility in Prague (described below). This allowed us to analyze the behavior of almost 90,000 residents - much higher number than alternative studies, which typically rely on on-site surveys with hundreds of participants. We studied multiple properties of the model output in order to reveal which factors are relevant for the daily pedestrian activity patterns.

\subsection{Model description}

The database of daily schedules and trips of synthetic Prague residents that was used for our analysis was generated by the multi-agent activity-based simulation model of Prague and Central Bohemian Region introduced in [16].

In contrast to traditional four-step models of transport demand [17], which use trips as the fundamental modeling unit, activity-based models employ so-called activities (e.g. work, shop, sleep) and their sequences to represent transport-related behavior of the population. Travel is then a derived demand occurring due to the necessity of the residents (agents) to satisfy their needs through activities performed at different places at different times. These activities are arranged in time and space into sequential (daily) schedules and are interconnected by trips, each consisting of one or multiple legs with specific mode of transport, route and timing. 
Trip origins, destinations and times are endogenous outcomes of activity scheduling. The activity-based approach considers individual trips in context and therefore produces more realistic trip chains.

The computational model used in this work covers a typical work day in Prague and Central Bohemian Region. The population of over 1.3 million is modeled by the same number of autonomous, self-interested agents, whose behavior is influenced by demographic attributes, current needs, context and cooperation.

Agents' decisions are implemented using artificial intelligence and machine learning methods (e.g. neural networks, genetic algorithms or decision trees) and trained beforehand using multiple real-world data sets, including census data or travel diaries and similar transportation-related surveys.

Planned activity schedules are subsequently simulated and calibrated on a supercomputer, and finally, their temporal, spatial and structural properties are validated against additional historic real-world data (origin-destination matrices and surveys) using six-step validation framework VALFRAM [18].

The model generates over 1.3 million daily schedules containing over 3 million trips per one 24-hour scenario. For our analysis, we selected a sample of 89,48 schedules of the agents who travel to work while using public transport for at least one of their trips.

\subsection{The sample description}

From the agent-based mobility model of Prague, we chose a sample of 89,949 agents with a specific mobility pattern. The agents regularly commute using public transport. The agents who don't commute to work (home-office workers, unemployed) or who commute by car were not included in this study. We also excluded all the student agents (younger than 21 years) and retired agents (older than 65 years, see Fig. 1). The number of suitable agents was finally reduced to 88,547 . The study focused on the daily pedestrian routine "residence work" and its influence on the overall walking activity per day. We focused on the relationship between the necessary and voluntary physical activity on a daily basis. From the mobility model, we retrieved the distance walked to the public transport stop, the distance walked from a different stop to the workplace, total distance walked during the day, the GPS coordinates of the residence, the financial income of the agent, the marital status, gender of the agent and highest reached education degree. Financial income of the agent is specified as a net monthly money income of the household divided uniformly among all the household members (converted by exchange rate $1 €=26.11 \mathrm{CZK}$ ).

\subsection{Observed factors}

Pedestrian activity (PA) of a selected category of agents for a day is an average value of all agents who belong to the selected category. The value is calculated as:

$$
\frac{\sum_{i=1}^{n_{s e l}} d_{s e l}}{n_{\text {sel }}}
$$

where $d_{\text {sel }}$ is a length of the trajectory walked for a day by an agent in the selected group, and $n_{\text {sel }}$ is the number of agents in selected group. The equation was also used for pedestrian activity constituted by walking from the residence to workplace and backwards.

Percentage of the walking activity recommendations (WAR) fulfilling agents in selected group was calculated as:

$$
\frac{n_{\text {act sel }}}{n_{\text {sel }}} \times 100
$$


where $n_{\text {act sel }}$ is a number of walking activity recommendations fulfilling agents in selected group, $n_{\text {sel }}$ is the number of agents in selected group.

\section{RESULTS}

\subsection{Daily pedestrian activity}

Average pedestrian activity (PA) of Prague resident represented by an agent, is 3,106 m per day. This constitutes only $47 \%$ of the walking activity recommendations of 10000 steps for a day $(6,600 \mathrm{~m})$ - less than half of the recommended pedestrian activity. Only $2.4 \%$ of the agents meet this public health recommendation. Using linear regression model, we proved on $5 \%$ level of significance that the percentage of active subject decreases by increasing age. On average, it decreases very slightly by $0.01 \%$ for each year of age.

We also found certain coherence between the average pedestrian activity and the activity constituted by walking from the residence to the workplace and backwards. This daily routine trajectory makes up for $85.4 \%$ of daily PA on average. Only $14.6 \%$ of walking activity for a day is not connected to the daily commuting routine (see Fig. 2).

Using two sample Welch t-test we proved on 0.05 level of significance that the true ratio of residence-workplace working doesn't differ from the observed mean by more than 0.25 $\%$. Using the t-test for the significance of a parameter in linear regression model we proved on all significance levels that daily walking distance decreases with increasing age. On average, it decreases by 3.96 meters for each year of age. It is slight decrease and we can prove it only because of the fact we work with quite large dataset. However, no linear dependence of resident-workplace walk and the age of the subject has been proved (p-value of the test of significance of the regression parameter was almost 0.3 ).

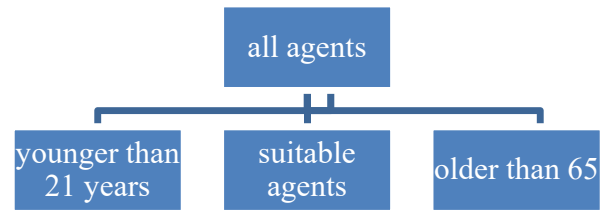

Figure 1: Structure of the sample, age separation.

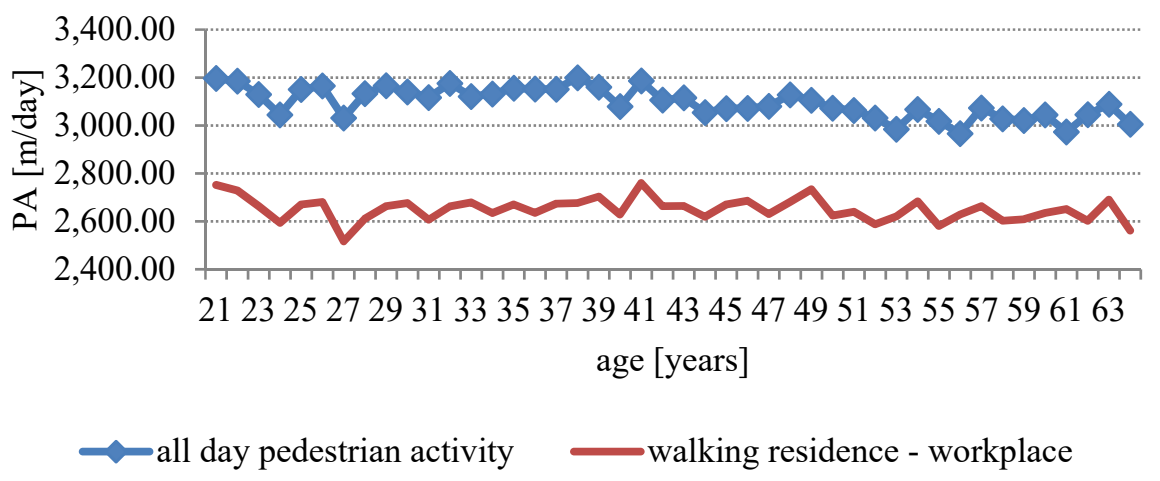

Figure 2: Average daily PA according age. 


\subsubsection{PA according age}

The difference between the daily pedestrian activity was not apparently connected to the agent's age. The average numbers were slightly lower with increasing age after 40 years, but the difference is small and irregularly distributed (see Fig. 2). The pedestrian activity would be possibly lower with higher age, but the participant agents are limited by the age of retirement. The WAR is met in $2.16 \%-2.62 \%$ of the agent population.

Using two sample Welch t-test we proved on all levels of significance that men walk more than women, 95\% confidence interval for the difference between true means of the covered daily distances equals $(132 \mathrm{~m}, 172 \mathrm{~m})$. However, there are no gender difference between residence-workplace covered distances (similarly to the fact that this distance doesn't even depends on age). For both genders, we can prove decreasing activity with increasing age, by $3.87 \mathrm{~m} /$ day for each year of age among the male population and by 4.07 $\mathrm{m} /$ day for each year of age among the female population.

\subsubsection{Relation between PA and gender}

The difference in pedestrian activity between male and female agents was noticeable. Female agents walked $3,021 \mathrm{~m}$ per day on average, compared to the average of $3,172 \mathrm{~m}$ walked by males. The female average pedestrian activity is therefore approximately $4,8 \%$ lower (see Fig. 4). Only $2 \%$ of female agents met the WAR, while the percentage in male population higher - approximately $2.7 \%$.

\subsubsection{Relation between PA and financial income}

For this analysis, we divided the agents into five groups according to their financial income. The income groups were calculated in CZK and converted to $€$. The difference between the group with the lowest and highest PA was 10\% (see Fig. 5). Surprisingly, the lowest PA was found in the group with the income of $1340 €-1720 €$ and the highest PA in the group with the income of $1,720 €$ and more. The difference between other income groups is not significant (see Fig. 5(a)).

The highest income group $(1,720 €$ and more) also contains the highest percentage of active agents. Specifically, 3.7\% of the agents in this income group fulfill the WAR. The percentage of agents meeting the WAR (see Fig. 5(b)) is similar in remaining income groups.

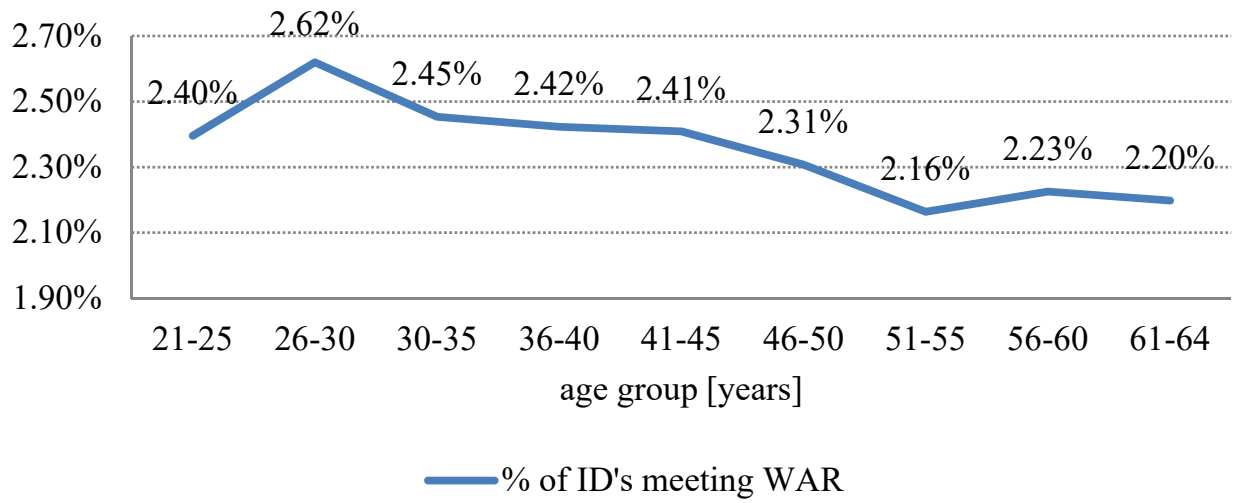

Figure 3: Active population according age. 
By using Welch-Anova test we prove on all significance levels the difference between pedestrian activity by the income group. Searching for the groups, which caused the overall difference, only no difference between the activity of the income group 3 and the income group 5 has been proven, all other groups are significantly differently active. If we order groups by average income, they are mixed together $(5,3,1,2,4$ by descending order) so no global conclusion about the influence of higher income group on the activity is obvious. However, if when we use income as a number (instead of the income group) as the independent variable and focus on dependence of it on the daily activity, we can prove it is statistically relevant using the linear regression model.

However, if when we use income as a number (instead of the income group) as the independent variable and focus on dependence of it on the daily activity, activity decreases on average by $3.21 \mathrm{~m}$.

\subsubsection{Relation between PA and marital status}

Next, we analyzed the agents in the following categories: single, married, divorced, widowed. Firstly, we analyze significance of the influence of the gender on the pedestrian activity, among all four categories of marital statuses. We use two sample Welch t-test. Statistical significance of the difference on the significance level $5 \%$ has been proved for all four categories.

We analyzed also significance of the influence of the gender on the percentage of active subjects. We conclude that it is statistically significant except the widowed subject where no statistically significant result can be stated.

Then we analyze influence of the marital status on the pedestrian activity and on percentage of active subjects, using Welch-Anova test. No differences of the pedestrian activity influenced by the marital status were proven as statistically relevant. Also, no difference of the percentage of active subject was discovered. These results sustain even if we analyze male population and female population separately.

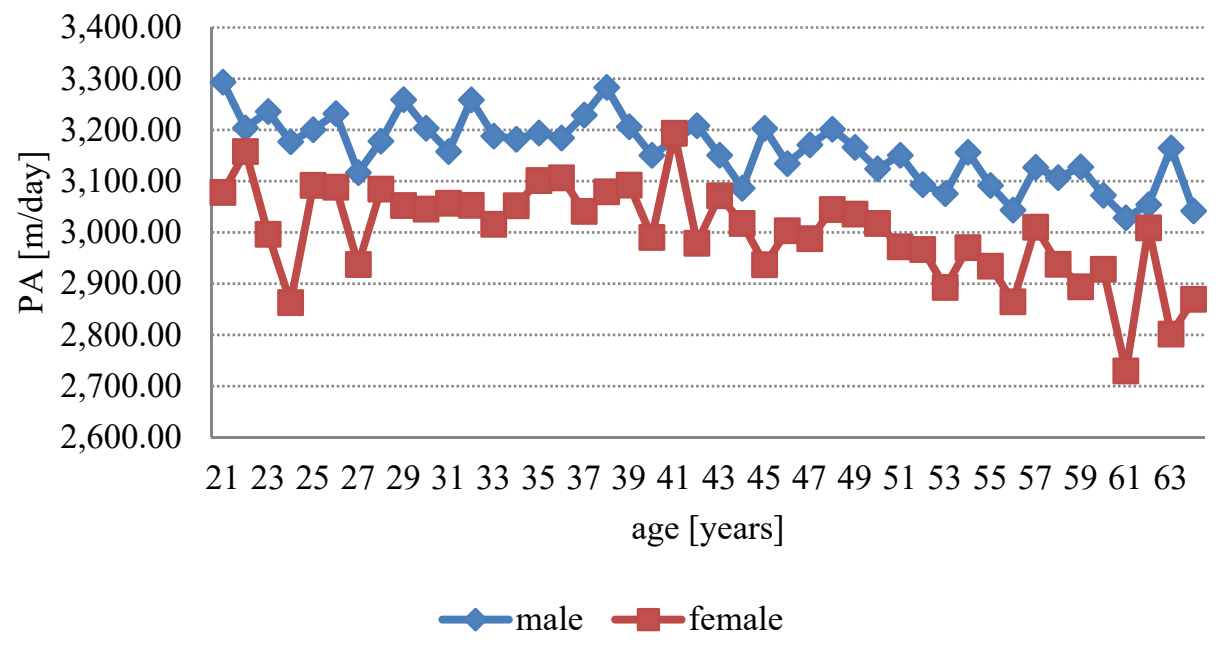

Figure 4: PA according gender and age. 


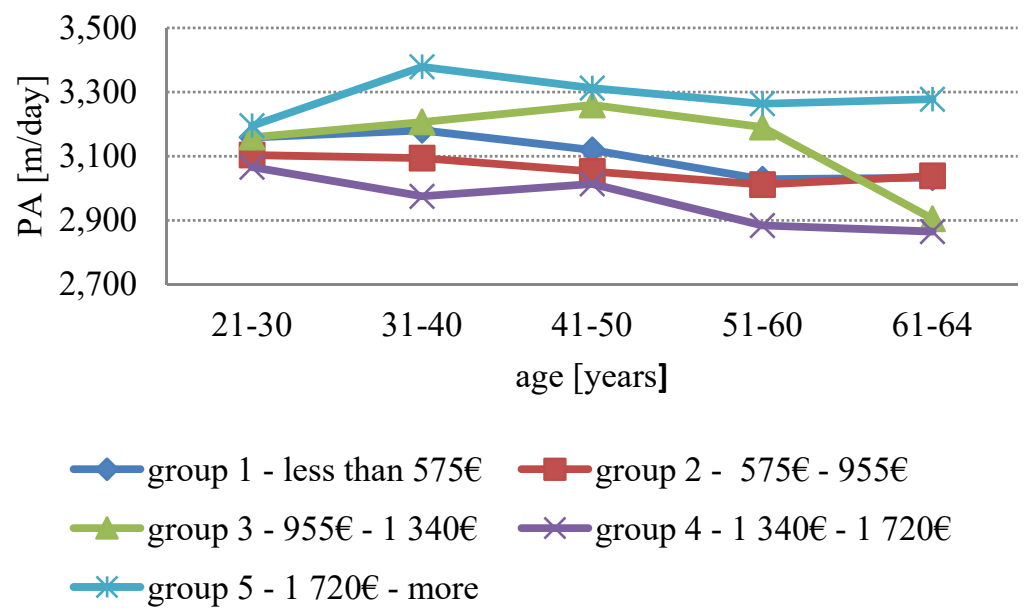

(a)

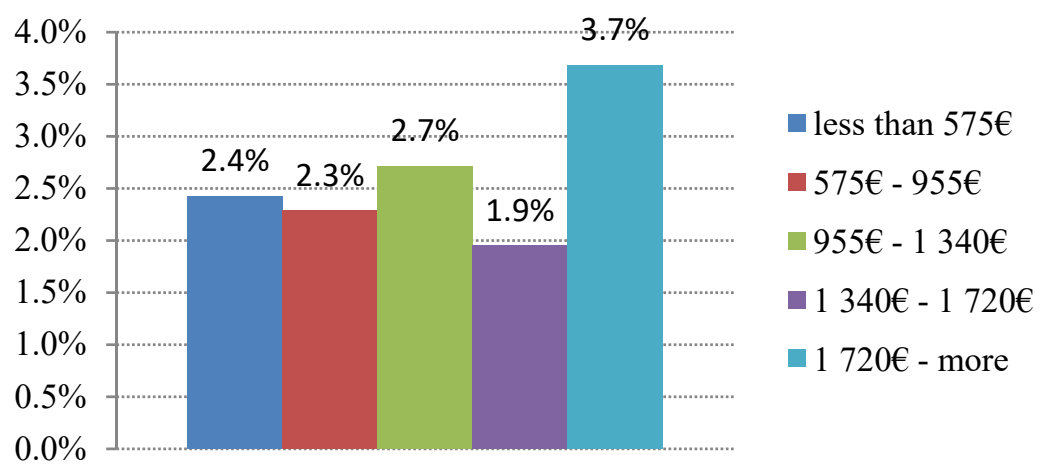

(b)

Figure 5: (a) PA according income group and age; (b) Percentage of WAR fulfilling agents according income group.

\subsubsection{Relation between PA and education}

Highest achieved degree of education seems to have a limited influence on the PA (See Fig. 7). We analyze significance of the influence of the gender on the PA, among all five categories given by the education level. We use two sample Welch t-test. Statistical significance of the difference has been proved for all five categories, on all levels of significance.

We conclude that the differences between average activity, given by the education, is significant on all levels of significance. We proved that higher education is connected with lower pedestrian activity. Searching for the groups causing the overall difference, we conclude that there are three clusters of education levels.

First of them is composed by the groups of the subjects with secondary education without final exam and with basic education, second one is composed only by the group with secondary education with final exams and the third cluster is composed by the remaining 


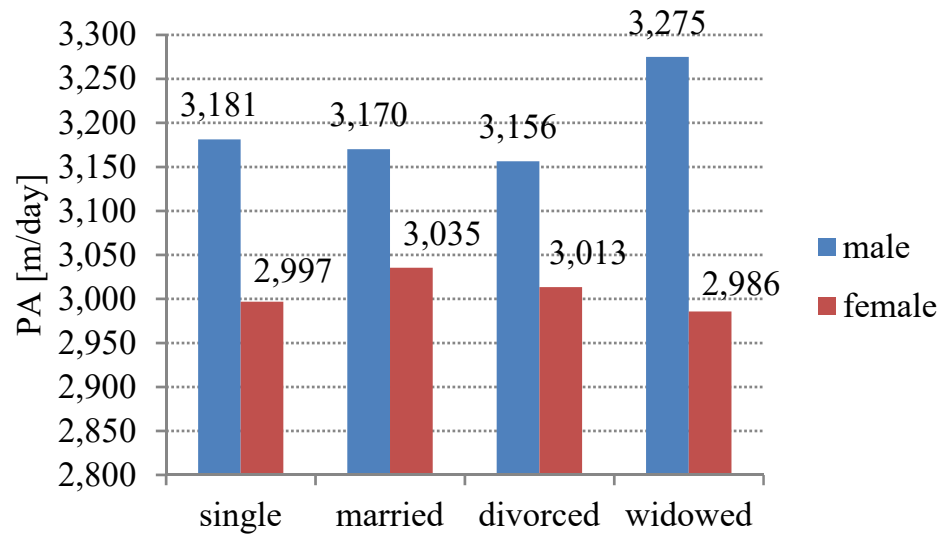

(a)

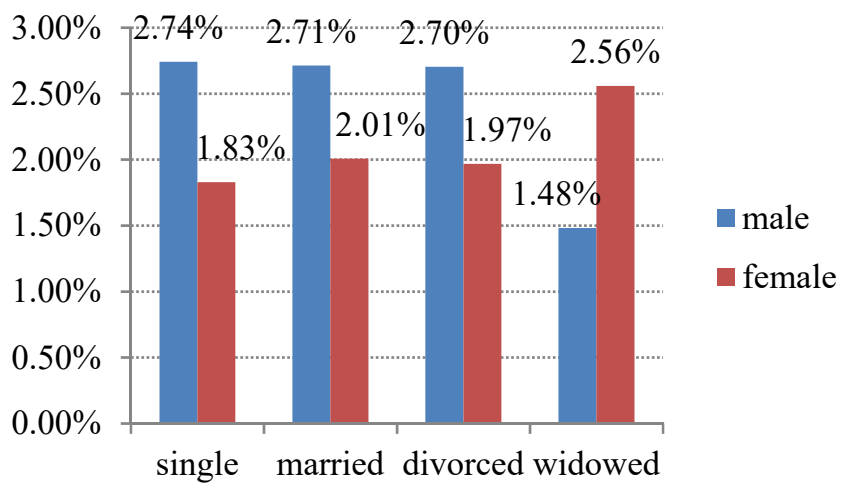

(b)

Figure 6: (a) PA according marital status; (b) Percentage of WAR fulfilling agents according marital status.

groups of higher professionals and university graduates. The clusters differ significantly, the groups in one cluster don't.

Among male population there is also significant difference between PA given by the level of education. Higher education is connected with lower pedestrian activity, as in the overall case. Even the pedestrian activity of the female population is significantly differed by the level of education. There are two clusters of groups causing the difference. First of them is composed by the groups with basic and secondary education. Second one is composed by groups higher professionals and university graduates.

Even the pedestrian activity of the female population is significantly differed by the level of education. There are two clusters of groups causing the difference. First of them is composed by the groups with basic and secondary education. Second one is composed by groups higher professionals and university graduates.

We analyzed also significance of the influence of the education on the percentage of active subjects. We conclude that it is statistically significant except the group of higher 
professionals where no statistically significant result can be stated, due to the law of small numbers.

Then we analyze influence of the education on the percentage of active subjects, using Welch-Anova test. No differences of the percentage of active subject given by the education level were proven as statistically relevant. This result sustains even if we analyze male population and female population separately.

\section{DISCUSSION}

The pedestrian activity (PA) among the residents of Prague represented by modeled agents is low. Only $2.16 \%-2.66 \%$ of population meets the WAR and can be considered as physical active. However, this study did not consider additional physical activities of the agents, such as sports and other means of transport demanding physical activity (cycling).

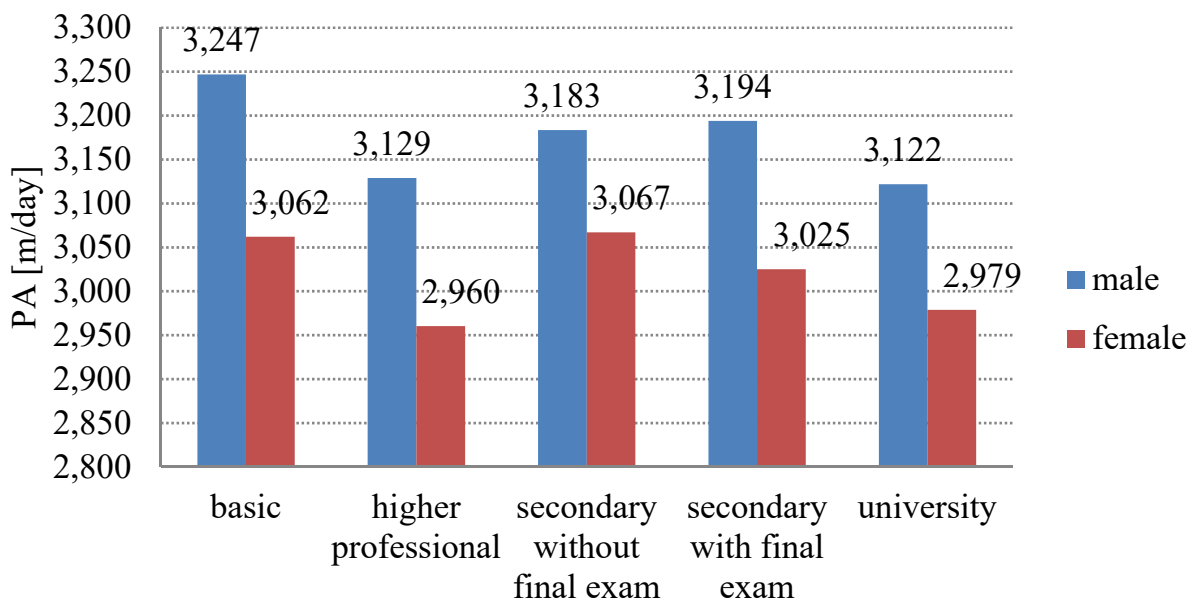

(a)

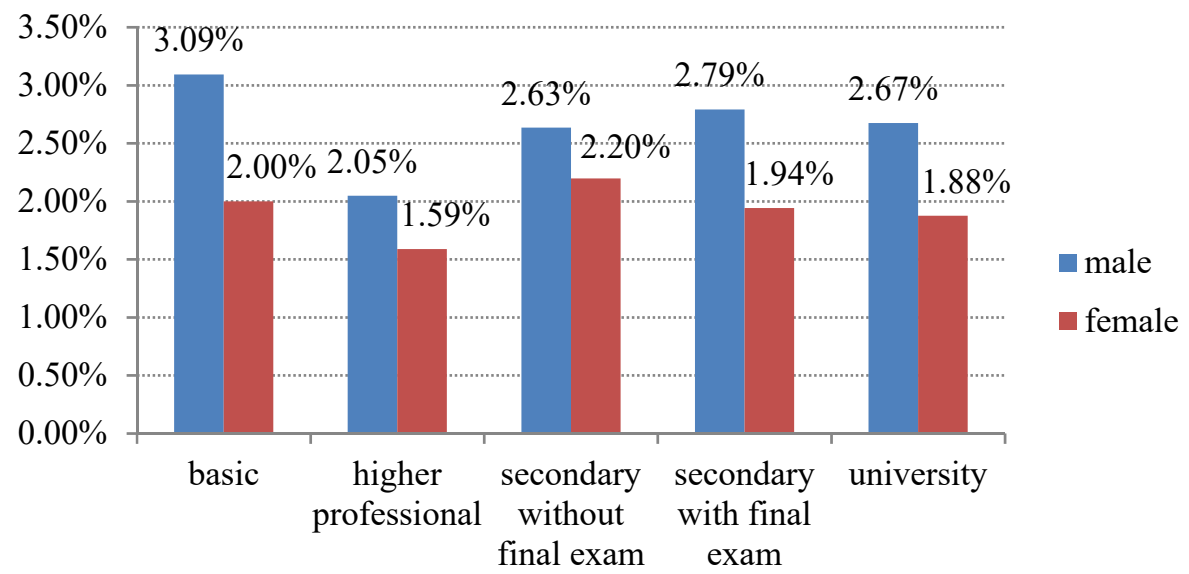

(b)

Figure 7: (a) PA according educational degree; (b) Percentage of WAR fulfilling agents according educational degree. 
Walking activity apart from the PA related the daily commuting routine is only $14,7 \%$ of the daily PA. Modeled agents tend to choose the shortest way to the destination. In reality, there are additional factors that influence the choice of the pedestrian route, such as quality of public space and pedestrian roads (19) (13), presence of the green vegetation, pollution along the frequented highways or presence of a shade in the summer (20). This can be addressed by using the exact trajectories from the GPS tracking, but unfortunately, it is currently hard to gather this kind of data for such a big population sample.

\subsection{Influence factors}

The difference in PA between the highest income group (1,720€ and more) can be caused by the location of their residence. The highest income group usually lives in residential quarters with low urban density, where public transport stops have bigger spacing. The second highest income group $(1,340 €-1,720 €)$ could be located close to public transport stations in denser urban structures, because the prices of the apartments and rents are higher than in the parts farther away from the public transport.

Marital status seems to have a negligible influence on the PA. It is surprising (and even slightly suspicious) that widowed males seemingly show bigger pedestrian activity with lower percentage of active subject, in comparison to widowed females. This discrepancy however results from the law of small numbers - we have only 280 widowed males in the dataset.

\section{CONCLUSION}

This study demonstrates a stable relationship between general daily pedestrian activity and the PA related specifically to commuting to the workplace. The PA connected to necessary daily routine constitutes $85.4 \%$ of total PA, while the non-routine PA makes up for only $14.6 \%$. This ratio is slightly influenced by the age of the population - the non-routine PA is decreasing with age, but the PA related to commuting to workplace remains.

We have shown the difference in PA among male and female residents. The PA of females was approximately $4.8 \%$ lower the PA of males. Similarly, the ratio of females meeting WAR was lower.

We detected a certain impact of the financial income on the PA. The group with the highest income evinced the highest average PA, while PA values of the group with the second highest income were lowest.

Noticeable was also the impact of the education. The difference between agents with different education were statistically relevant in groups, but we didn't prove dependency of PA of agents is coherently increasing or decreasing with the level of reached education degree.

\section{ACKNOWLEDGEMENT}

We thank Martin Dungl for the help with statistical computation.

\section{REFERENCES}

[1] Gehl, J. \& Svarre, B., How to Study Public Life, Island Press: Washington DC, pp. 4648, 2013.

[2] CIAM's The Athens Charter (1933), Modernist architecture, Online. https://modernistarchitecture.wordpress.com/2010/11/03/ciam $\%$ E2\%80\%99s- $\%$ E2\% 80\%9Cthe-athens-charter\%E2\%80\%9D-1933/. Accessed on: 6 Jun. 2017.

[3] Jacobs, J., Life and Death of Great American Cities, Random House: New York, 1993. 
[4] Kohl 3rd, H.W. et al., The pandemic of physical inactivity: global action for public health. Lancet, 380, pp. 294-305, 2012.

[5] Nakládalová, M., Sovová, E., Ivanová, K, Kaletová, M., Lukl, J. \& Fialová, J., Risk factors for cardiovascular diseases in physicians. Biomed Pap Med Fac Univ Palacky, 149(2), pp. 293-295, 2005.

[6] Health statistics yearbook of the Hl. m. Praha Region 2013, Institute of Health Information and Statistics of the Czech Republic: Prague, 2014.

[7] Iwane, M. et al., Walking 10,000 Steps/Day or More Reduces Blood Pressure and Symphatheic Nerve Activity in Mild Essential Hypertension. Hypertens Res, 6(23), pp. 573-580, 2000.

[8] Tudor-Locke, C. \& Bassett, D.R. Jr., How many steps/day are enough? Sports Med., 34, pp. 1-8, 2004.

[9] Nelson, M.E., Rejeski, W.J., Blair, S.N., Duncan, P.W. \& Judge, J.O., Physical Activity and Public Health in Older Adults: Recommendation from the American College of Sports Medicine and the American Heart Association. Circulation, 9(116), pp. 10941105, 2007.

[10] Saelens, B.E.F., Sallis, J. \& Frank, L.D., Environmental Correlates of Walking and Cycling: Findings from the Transportation, Urban Design, and Planning Literatures. Environment and Physical Activity, 2(25), pp. 80-91, 2003.

[11] Soni, N. \& Soni, N., Benefits of pedestrianization and warrants to pedestrianize an area. Land Use Policy, 57, pp. 139-150, 2016.

[12] Brown, D.K.., Barton, J.L., Pretty, J. \& Gladwell, V.F., Walks4Work: Assessing the role of the natural environment in a workplace physical activity intervention. Environment \& Health Scandinavian Journal of Work, 2014.

[13] Handy, S.L., Boarnet, M.G., Ewing, R. \& Killingsworth, R.E., How the Built Environment Affects Physical Activity - Views from Urban Planning. American Journal of Preventive Medicine, 23(2), pp. 64-73, 2002.

[14] The National Institute of Public Health (NIPH), http://www.szu.cz/uploads/documents/ obi/CAV/6.CAV_3_Zmeny_telesnych_proporc.pdf. Accessed on: 6 Jun. 2017.

[15] Čtyroký, M.J., section director of Spatial Information Section, Prague Institute of Planning and Development, Prague, 2017.

[16] Čertický, M., Drchal, M., Cuchý, M. \& Jakob, M., Fully Agent-based Simulation Model of Multimodal Mobility in European Cities. Models and Technologies for Intelligent Transportation Systems, 2015.

[17] McNally, M.G., "The four-step model”. Handbook of Transport Modelling, 2nd Edition, Emerald Group Publishing Limited: West Yorkshire, pp. 35-53, 2007.

[18] Drchal, J., Čertický, M. \& Jakob, M., Data Driven Validation Framework for MultiAgent Activity-based Models. Multi-Agent Based Simulation XVI. MABS 2015, Springer International Publishing: Cham, 2015.

[19] Mäki-Opas, T.E. et al., The contribution of travel-related urban zones, cycling and pedestrian networks and green space to commuting physical activity among adults $-\mathrm{a}$ cross-sectional population-based study using geographical information systems. BMC Public Health, BioMed Central: London, 2016.

[20] Rahman, N.A., Shamsuddin, S. \& Ghani, I., What Makes People Use the Street? Towards a livable urban environment in Kuala Lumpur city centre. Procedia - Social and Behavioral Sciences, 170, pp. 624-632, 2015.

[21] Guessous, I. et al., A comparison of the spatial dependence of body mass index among adults and children in a Swiss general population. Nutrition \& Diabetes, 111(4), 2014. 